\title{
Treadmill Exercise Improves Motor Function and Short-term Memory by Enhancing Synaptic Plasticity and Neurogenesis in Photothrombotic Stroke Mice
}

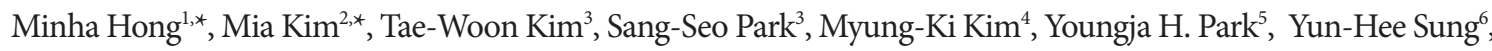 \\ Mal-Soon Shin ${ }^{4}$ \\ ${ }^{1}$ Department of Psychiatry, Myongji Hospital, College of Medicine, Hanyang University, Goyang, Korea \\ ${ }^{2}$ Department of Cardiovascular Neurologic Disease (Stroke Center), College of Korean Medicine, Kyung Hee University, Seoul, Korea \\ ${ }^{3}$ Department of Physiology, College of Medicine, Kyung Hee University, Seoul, Korea \\ ${ }^{4}$ School of Global Sport Studies, Korea University, Sejong, Korea \\ ${ }^{5}$ Metabolomics Laboratory, College of Pharmacy, Korea University, Sejong, Korea \\ ${ }^{6}$ Department of Physical Therapy, College of Health Sciences, Kyungnam University, Changwon, Korea
}

Purpose: Thrombotic stroke is a type of ischemic stroke characterized by motor dysfunction and memory impairments. In the present study, the effect of treadmill exercise on motor function and short-term memory was evaluated in relation with synaptic plasticity in the mice with photothrombotic stroke.

Methods: Photothrombotic stroke was induced by cortical photothrombotic vascular occlusion. The mice in the treadmill exercise groups performed running on a motorized treadmill for 28 days. Motor function was determined using rota-rod test and foot fault test. Step-through avoidance task was conducted to evaluate short-term memory. Immunohistochemistry for 5-bromo-2'-deoxyuridine and doublecortin was conducted to detect new cell generation. Postsynaptic density protein 95, synaptophysin, brain-derived neurotrophic factor (BDNF), and tyrosine kinase B receptor (TrkB) were determined using western blot. The number of dendritic spines was determined using Golgi stain.

Results: Treadmill exercise improved motor function and short-term memory in mice with the photothrombotic stroke. The infarct size was reduced and the number of dendritic spines and expression of postsynaptic density protein 95 and synaptophysin in the peri-infarct cortex and hippocampus were increased by treadmill exercise in photothrombotic stroke mice. Treadmill exercise enhanced neurogenesis through increasing the expression of the hippocampal BDNF and TrkB in photothrombotic stroke mice.

Conclusions: Treadmill exercise improved motor function and short-term memory through increasing synaptic plasticity and neurogenesis in photothrombotic stroke mice. Treadmill exercise can be used as an effective treatment strategy to improve brain function related to stroke.

Keywords: Photothrombotic stroke; Exercise; Short-term memory; Synaptic plasticity

- Fund/Grant Support: This study was supported by the Ministry of Education of the Republic of Korea and the National Research Foundation of Korea (NRF-2018S1A5A8026767).

- Research Ethics: We obtained the approval from the Kyung Hee University Institutional Animal Care and Use Committee (Seoul, Korea)

(KHUASP [SE]-19-172).

- Conflict of Interest: No potential conflict of interest relevant to this article was reported.

Corresponding author: Mal-Soon Shin (iD https://orcid.org/0000-0002-7462-2211 College of Culture and Sports, Division of Global Sport Studies, Korea University, 2511 Sejong-ro, Sejong 30019, Korea

E-mail: malsoon@korea.ac.kr / Tel: +82-044-860-1365 / Fax: +82-044-860-1589 Submitted: March 25, 2020 / Accepted after revision: April 28, 2020

${ }^{*}$ Minha Hong and Mia Kim contributed equally to this study as co-first authors.
This is an Open Access article distributed under the terms of the Creative Commons Attribution Non-Commercial License (http://creativecommons.org/licenses/by-nc/4.0/) which permits unrestricted non-commercial use, distribution, and reproduction in any medium, provided the original work is properly cited. 


\author{
- HIGHLIGHTS \\ - Thrombotic stroke is a type of ischemic stroke characterized by motor dysfunction and memory impairments. \\ - Treadmill exercise reduced infarct size and increased number of dendritic spines and expression of PSD-95 and synaptophysin in photo- \\ thrombotic stroke mice. \\ - Treadmill exercise improved motor function and short-term memory in mice with the photothrombotic stroke.
}

\section{INTRODUCTION}

Ischemic stroke is the most common type of stroke, and this disease is induced by a decrease in brain blood supply caused by permanent or temporary artery occlusion in cerebral bloodstream $[1,2]$. The thrombotic stroke of the brain is a type of neurological stroke caused by thrombus formation, resulted in neuronal death and neurological disorders [3]. The photothrombotic stroke is a commonly used stroke model that causes ischemic infarction in certain areas of the cortex [4].

Brain synaptic plasticity has emerged as an effective treatment for stroke [5]. Synaptic plasticity is thought to contribute to learning and memory through changes in synaptic strength [6]. Postsynaptic density protein 95 (PSD-95) and synaptophysin are important indicators of synaptic plasticity [7]. In addition, synaptophysin, also known as the major synaptic vesicle protein p38-1 [8], is a protein that in humans is encoded by the SYP gene. As a presynaptic marker, synaptophysin is mainly expressed in neurons.

Neurogenesis occurs throughout adulthood in difference areas of the brain. Adult hippocampal neurogenesis is closely implicated in hippocampal neuroplasticity and memory formation [9]. Damage to the neurogenesis in the hippocampal dentate gyrus impairs memory formation and performance, and the severity of this damage is correlate with the degree of damage to the granule cell precursors [10]. This neurogenesis is influenced and regulated by diverse neurotrophic factors, including brain-derived neurotrophic factor (BDNF) and nerve growth factor [11]. BDNF regulates the action of increasing cell proliferation of exercise [12], and this action of BDNF is mediated primarily by the tyrosine kinase $B$ receptor (TrkB) [13] .

Physical exercise can improve neurological symptoms by preventing neuronal loss from brain ischemic insults, and exercise has been proposed as a possible therapeutic adjunct to cerebral ischemia [14-16]. Ding et al. [16] reported that regular treadmill exercise alleviated neurologic deficits and reduced infarct volume in the cortex and striatum after ischemia. However, the effect of exercise on motor function and short-term memory in association with synaptic plasticity in thrombotic stroke has not been established.

In this study, we designed to evaluate whether treadmill exercise improves motor function and short-term memory in association with synaptic plasticity using the mice with photothrombotic stroke. For this study, motor function was assessed using the rota-rod test and foot fault test, and short-term memory was evaluated using the step-through avoidance task. Cell proliferation was detected using immunohistochemistry for 5-bromo-2'-deoxyuridine (BrdU) and doublecortin (DCX). Expression of PSD-95, synaptophysin, BDNF, TrkB was measured by western blot, and Golgi staining for dendrite spine measurement was also performed.

\section{MATERIALS AND METHODS}

\section{Animals}

For this experiment, C57BL/6 male mice at 8 weeks of age (weight $25 \mathrm{~g}, \mathrm{n}=40$ ) were used. The experimental process carried out the guidelines of animal care by the National Institutes of Health and Korea Medical Center. We obtained the approval from the Kyung Hee University Institutional Animal Care and Use Committee (KHUASP [SE]-19-172). Mice were classified into the sham-operation group, sham-operation and treadmill exercise group, the photothrombotic stroke-induction group, and the photothrombotic stroke-induction and treadmill exercise group ( $\mathrm{n}=10$ in each group). All the mice were injected intraperitoneally with $50-\mathrm{mg} / \mathrm{kg}$ BrdU (Sigma Chemical Co., St Louis, MO, USA). The injection was given 30 minutes before the starting of the treadmill exercise once a day, and the injection was continued for 7 days.

\section{Photothrombotic Stroke}

Photothrombotic stroke was induced using cortical photothrombotic vascular occlusion with reference to the previously described method [17]. Intraperitoneal injection of Zoletil 50 (10 mg/kg; Vibac Laboratories, Carros, France) was used to anesthetize the mice. The head was fixed in a stereotaxic frame, then the scalp was incised to expose the skull. A fiber-optic bundle of a cold light source (KL 1500 LCD; Carl Zeiss, Göttin- 
gen, Germany) with a 4-mm aperture was put close to the skull. And then irradiated with cold light for 15 minutes at a right lateral distance of $2 \mathrm{~mm}$ from the bregma after 5 minutes of rose bengal ( $100 \mu \mathrm{L}$ of $10 \mathrm{mg} / \mathrm{mL}$; Sigma-Aldrich, St. Louis, MO, USA) intraperitoneal injection. The mice in the sham-operation group underwent the same operation procedure without irradiation.

\section{Treadmill Exercise Protocol}

The mice in the treadmill exercise groups were submitted to treadmill running for daily 30 minutes once a day for 28 days, starting one day after induction of photothrombotic stroke. The load of exercise consisted of running at the initial speed of $3 \mathrm{~m} /$ min for the first 5 minutes, $5 \mathrm{~m} /$ minute for the next 5 minutes, and $8 \mathrm{~m} /$ minute for the last 20 minutes with the $0^{\circ}$ inclination [18].

\section{Rota-Rod Test}

Rota-rod test was performed to evaluate the motor balance and coordination with reference to the previously described method [19]. The mouse was placed on a rotating drum on the rota-rod (3-cm diameter, 8.5-cm width). By measuring how long it lasts on a rotating drum, it measures the motor's ability to sense equilibrium. The velocity of the rod was increased from $4 \mathrm{rpm}$ to $20 \mathrm{rpm}$ over 5 minutes and the latency to fall is recorded. The maximum time to fall was limited to 180 seconds.

\section{Foot Fault Test}

The foot fault test is a tool for determining locomotor function of central nervous system disorders in rodent model. A foot fault test was performed to evaluate the locomotor function as the previously described method [20]. The mouse was placed on a steel grid (bars $2.5 \mathrm{~mm}$ in diameter, spaced $1 \mathrm{~cm}$ apart). Each step that successfully placed the foot was recorded step by step. A paw slipping through the grate was considered a foot fault. The number of steps and faults was determined for each paw. Percentage of foot faults $=$ faults $/($ steps + faults $) \times 100 \%$.

\section{Step-Through Avoidance Task}

The step-through avoidance task was also used to measure short-term memory with reference to the previously described method [14]. The equipment consisted of 2 compartments (dark/light shuttle box) separated by a grid door. The dark compartment had stainless steel shock grid floor (0.5 mA). During the acquisition trial, the mouse was placed in the illuminated light compartment, and the door between compartments was opened after 60 seconds. The time taken for a mouse to enter the dark compartment was recorded. When the mouse entered the dark room, the door was closed and electrical foot shock was delivered to the floor grids for 3 seconds. After 24 hours, the time taken to enter the dark room without electrical shock was measured in the same manner as the acquisition trial. The latency was set to a maximum of 600 seconds.

\section{Infarct Size}

At the 28 days after induction of photothrombotic stroke, the mice were sacrificed, and then infarct size was analyzed using 2,3,5-triphenyltetrazolium chloride (TTC) staining. Brains were immediately removed and sectioned at $2 \mathrm{~mm}$ thick. Then sections were incubated with $2 \%$ TTC for 30 minutes at $37^{\circ} \mathrm{C}$, fixed in $4 \%$ paraformaldehyde. Image-Pro Plus image analyzer (Media Cyberbetics Inc., Silver Spring, MD, USA) was used to measure the infarct area. The infarct size was calculated by using following formula: $\%$ infarct volume $=($ infarct volume $/$ volume of the homolateral hemisphere) $\times 100 \%$.

\section{Golgi Stain Procedure}

Golgi stain was also used to analyze dendrite spines. The procedure followed FD Rapid GolgiStain protocol (FD NeuroTechnologies Inc., Columbia, MD, USA). Brains were sectioned on a cryostat with $100-\mu \mathrm{m}$ thickness and mounted on gelatin-coated microscope slides. The slides were cleared in xylene and cover-slipped with Permount mounting media (Thermo Fisher Scientific, Waltham, MA, USA). To measure spine density, periinfarct cortex and hippocampus from each animal were selected. Images were observed with a $100 \times$ oil immersion objective lens by Image-Pro Plus Image analyzer. The spine number was expressed as the number of spines $/ 10 \mu \mathrm{m}$.

\section{Tissue Preparation}

Mice were anesthetized using Zoletil 50 (10 mg/kg, intraperitoneally; Vibac Laboratories) and then prepared to separate brain tissue. Then the mice were transcardially perfused using by $50 \mathrm{mM}$ phosphate-buffered saline (PBS), and they are fixed by a freshly $4 \%$ paraformaldehyde in $100 \mathrm{mM}$ phosphate buffer $(\mathrm{pH}$, 7.4). After brains were removed, then brains were postfixed in the $4 \%$ paraformaldehyde overnight at $4^{\circ} \mathrm{C}$. For cryoprotection, brains were transferred into the $30 \%$ sucrose solution. Forty $\mu \mathrm{m}$ thick coronal sections were made using a freezing microtome (Leica, Nussloch, Germany). 


\section{Immunohistochemistry for BrdU}

For measuring proliferative neuronal cells in the hippocampal dentate gyrus, immunohistochemistry for BrdU was conducted with reference to the previously described method [13,21]. First, the sections were permeabilized by incubation with $0.5 \%$ Triton X-100 in PBS for 20 minutes, then pretreated in 50\% formamide- $2 \mathrm{X}$ standard saline citrate for 2 hours at $65^{\circ} \mathrm{C}$. The sections were denatured with $2 \mathrm{~N} \mathrm{HCl}$ for 30 minutes at $37^{\circ} \mathrm{C}$, and rinsed twice with $100 \mathrm{mM}$ sodium borate $(\mathrm{pH}, 8.5)$. Then, the sections were incubated with mouse monoclonal anti-BrdU antibody (1:600; Roche, Mannheim, Germany) at $4^{\circ} \mathrm{C}$ overnight. The next day, the sections were washed with PBS 3 times and incubated with the mouse biotinylated secondary antibody (1:200; Vector Laboratories, Burlingame, CA, USA) for 120 minutes. Counter-staining was conducted on the same sections using a mouse monoclonal antineuronal nuclei (NeuN) antibody (1:1,000; Chemicon International, Temecula, CA, USA) following BrdU staining. After dehydration through a gradient of ethanol, the slides were mounted with coverslips using Permount mounting media (Thermo Fisher Scientific).

\section{Immunohistochemistry}

According to a previously described method [13], DCX immunohistochemistry was conducted. The sections were incubated by goat anti-DCX antibody (1:500; Santa Cruz Biotechnology, Santa Cruz, CA, USA) overnight and next incubated by biotinylated goat secondary antibody (1:200; Vector Laboratories) for another 1 hour. Then, the bound secondary antibody was amplified with a Vector Elite ABC kit (1:200; Vector Laboratories). The $0.02 \%$ diaminobenzidine was used to visualize the antibody-biotin-avidin-peroxidase complex. After dehydration through a gradient of ethanol, the slides were mounted with coverslips using Permount ${ }^{\mathrm{TM}}$ mounting media (Thermo Fisher Scientific).

\section{Western Blotting}

According to a previously described method [13,21], western blot was conducted. From the peri-infarct cortex and hippocampus, total protein was extracted using lysis buffer containing $150 \mathrm{mM} \mathrm{NaCl}, 1 \mathrm{mM}$ phenylmethylsulfonyl fluoride, $1 \%$ Nonidet P40, 0.5\% deoxycholic acid, $0.1 \%$ sodium dodecyl sulfate (SDS), $100-\mathrm{mg} / \mathrm{mL}$ leupeptin, $50 \mathrm{mM}$ Tris- $\mathrm{HCl}$ (pH, 7.5). Protein was quantified using a Bradford protein assay (Bio-Rad, Hercules, CA, USA). Protein, $30 \mu \mathrm{g}$, was separated on SDS polyacrylamide gel electrophoresis. After electrophoresis, pro- teins were transferred to nitrocellulose membrane (GE Healthcare Life Sciences, Chicago, IL, USA). The membrane was blocked with skim milk, then membrane was incubated by mouse anti- $\beta$-actin antibody (1:1,000; Santa Cruz Biotechnology), rabbit anti-PSD-95 antibody (1:1,000; Abcam, Cambridge, UK), rabbit antisynaptophysin (1:1,000; Abcam), rabbit antiBDNF antibody (1:1,000; Alomone Labs, Jerusalem, Israel), and rabbit anti-TrkB antibody (Santa Cruz Biotechnology) at $4^{\circ} \mathrm{C}$ during overnight. After washing, species appropriate horseradish peroxidase-conjugated secondary antibodies were incubated for 1 hour. Band detection was performed using the enhanced chemiluminescence detection system (Santa Cruz Biotechnology).

\section{Data Processing}

By using the Image-Pro Plus image analyzer, the number of $\mathrm{Br}$ dU-positive cells and DCX-positive cells in the hippocampal dentate gyrus was calculated hemilaterally. The data were expressed as the number of cells per $\mathrm{mm}^{2}$ of the granular layer of the hippocampal dentate gyrus. Western blot bands were quantified by Image-Pro Plus image analyzer.

IBM SPSS Statistics ver. 21.0 (IBM Co., Armonk, NY, USA) was used for statistical analysis. The results were expressed as the mean \pm standard error of the mean. Significance among the groups was analyzed by 1-way analysis of variance test and followed by Duncan multiple range test. Significance was determined at $\mathrm{P}<0.05$.

\section{RESULTS}

\section{Motor Function}

Fig. 1 shows the latency of rota-rod test (panel A) and foot fault score of foot fault test (panel B). For the rota-rod test, induction of photothrombotic stroke reduced the latency to fall compared with the sham-operation group $(\mathrm{P}<0.05)$, while treadmill exercise enhanced the latency to fall compared with the photothrombotic stroke-induction group $(\mathrm{P}<0.05)$. For the foot fault test, induction of photothrombotic stroke reduced the foot fault score compared with the sham-operation group $(\mathrm{P}<0.05)$, while treadmill exercise enhanced the foot fault score compared with the photothrombotic stroke-induction group $(\mathrm{P}<0.05)$.

\section{Short-Term Memory}

Fig. 2 shows latency of the short-term memory in the stepthrough avoidance task. The induction of photothrombotic 

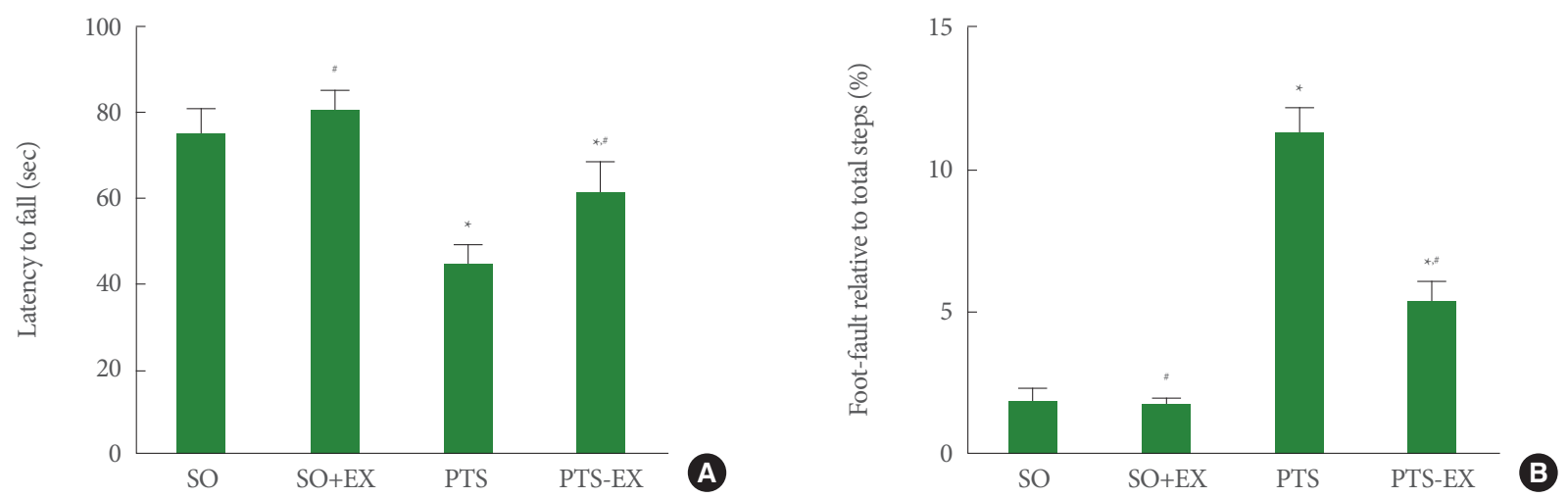

Fig. 1. Motor function in the rota-rod test (A) and the foot fault test (B). SO, sham-operation group; SO+EX, sham-operation and exercise group; PTS, photothrombotic stroke-induction group; PTS+EX, photothrombotic stroke-induction and exercise group. Data show mean \pm standard error of the mean. ${ }^{*} \mathrm{P}<0.05$ compared with the sham-operation group. ${ }^{*} \mathrm{P}<0.05$ compared with the photothrombotic stroke-induction group.

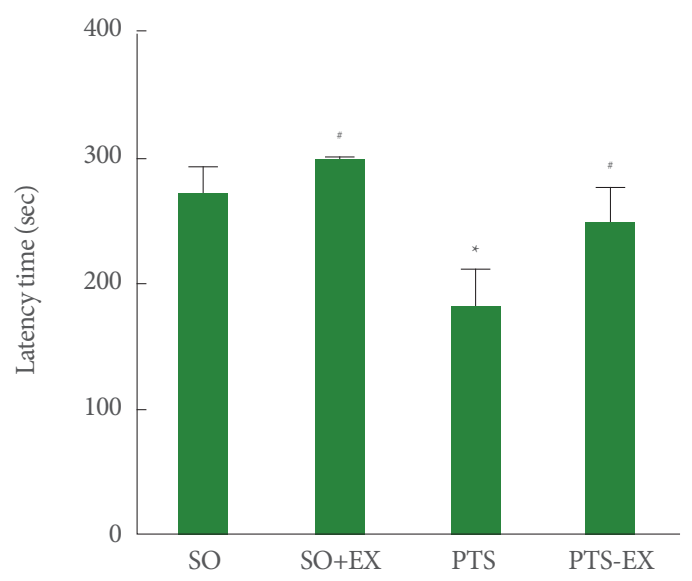

Fig. 2. Short-term memory in the step-through avoidance task. $\mathrm{SO}$, sham-operation group; $\mathrm{SO}+\mathrm{EX}$, sham-operation and exercise group; PTS, photothrombotic stroke-induction group; PTS+EX, photothrombotic stroke-induction and exercise group. Data show mean \pm standard error of the mean. ${ }^{\star} \mathrm{P}<0.05$ compared with the sham-operation group. ${ }^{*} \mathrm{P}<0.05$ compared with the photothrombotic stroke-induction group.

stroke reduced latency of the short-term memory compared with the sham-operation group $(\mathrm{P}<0.05)$, while treadmill exercise enhanced latency of the short-term memory compared with the photothrombotic stroke-induction group $(\mathrm{P}<0.05)$.

\section{Infarct Size}

Fig. 3 shows infarct size following photothrombotic stroke. The induction of photothrombotic stroke increased the infarct size compared with sham-operation group $(\mathrm{P}<0.05)$, while tread- mill exercise reduced the infarct size compared with the photothrombotic stroke-induction group $(\mathrm{P}<0.05)$.

\section{Dendritic Spines Number}

Fig. 4 shows dendritic spines in the peri-infarct cortex (panel A) and the hippocampal dentate gyrus (panel B). The induction of photothrombotic stroke reduced the number of dendritic spines in the peri-infarct cortex compared with the sham-operation group $(\mathrm{P}<0.05)$, while treadmill exercise enhanced the number of dendritic spines compared with the photothrombotic stroke-induction group $(\mathrm{P}<0.05)$. The induction of photothrombotic stroke reduced the number of dendritic spines in the hippocampal dentate gyrus compared with the sham-operation group $(\mathrm{P}<0.05)$, while treadmill exercise enhanced the number of dendritic spines compared with the photothrombotic stroke-induction group $(\mathrm{P}<0.05)$.

\section{PSD-95 and Synaptophysin Expression}

Fig. 5 shows the relative expression of PSD-95 and synaptophysin in the peri-infarct cortex (panel A) and hippocampal dentate gyrus (panel B). The induction of photothrombotic stroke reduced the relative expression of PSD-95 and synaptophysin in the peri-infarct cortex $(\mathrm{P}<0.05)$, while treadmill exercise enhanced the relative expression of PSD-95 and synaptophysin compared with the photothrombotic stroke-induction group $(\mathrm{P}<0.05)$. The induction of photothrombotic stroke reduced the relative expression of PSD-95 and synaptophysin in the hippocampal dentate gyrus $(\mathrm{P}<0.05)$, while treadmill exercise enhanced the relative expression of PSD-95 and synaptophysin 

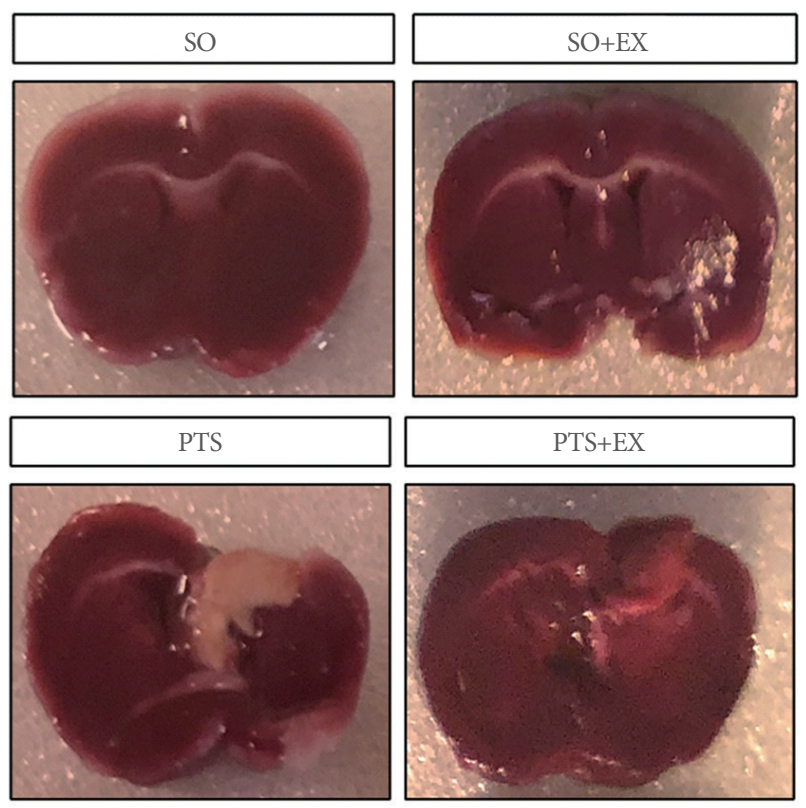

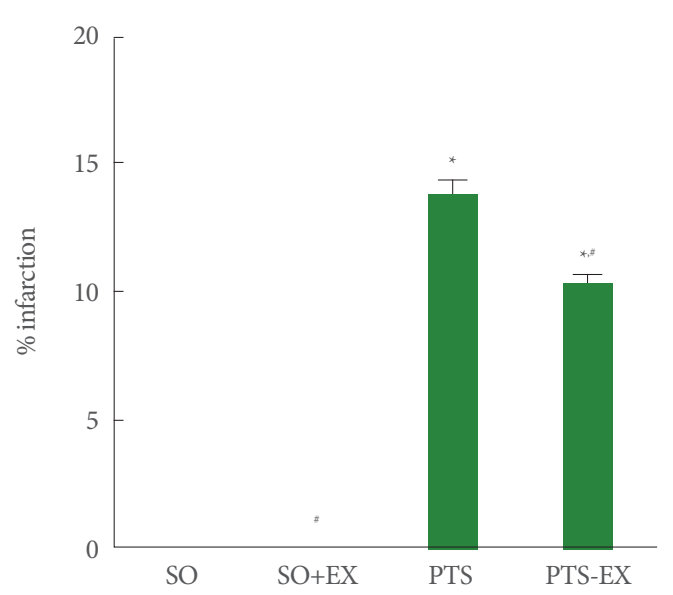

Fig. 3. Infarct size. SO, sham-operation group; SO+EX, sham-operation and exercise group; PTS, photothrombotic stroke-induction group; PTS+EX, photothrombotic stroke-induction and exercise group. Data show mean \pm standard error of the mean. ${ }^{\star} \mathrm{P}<0.05 \mathrm{com}$ pared with the sham-operation group. ${ }^{*} \mathrm{P}<0.05$ compared with the photothrombotic stroke-induction group.
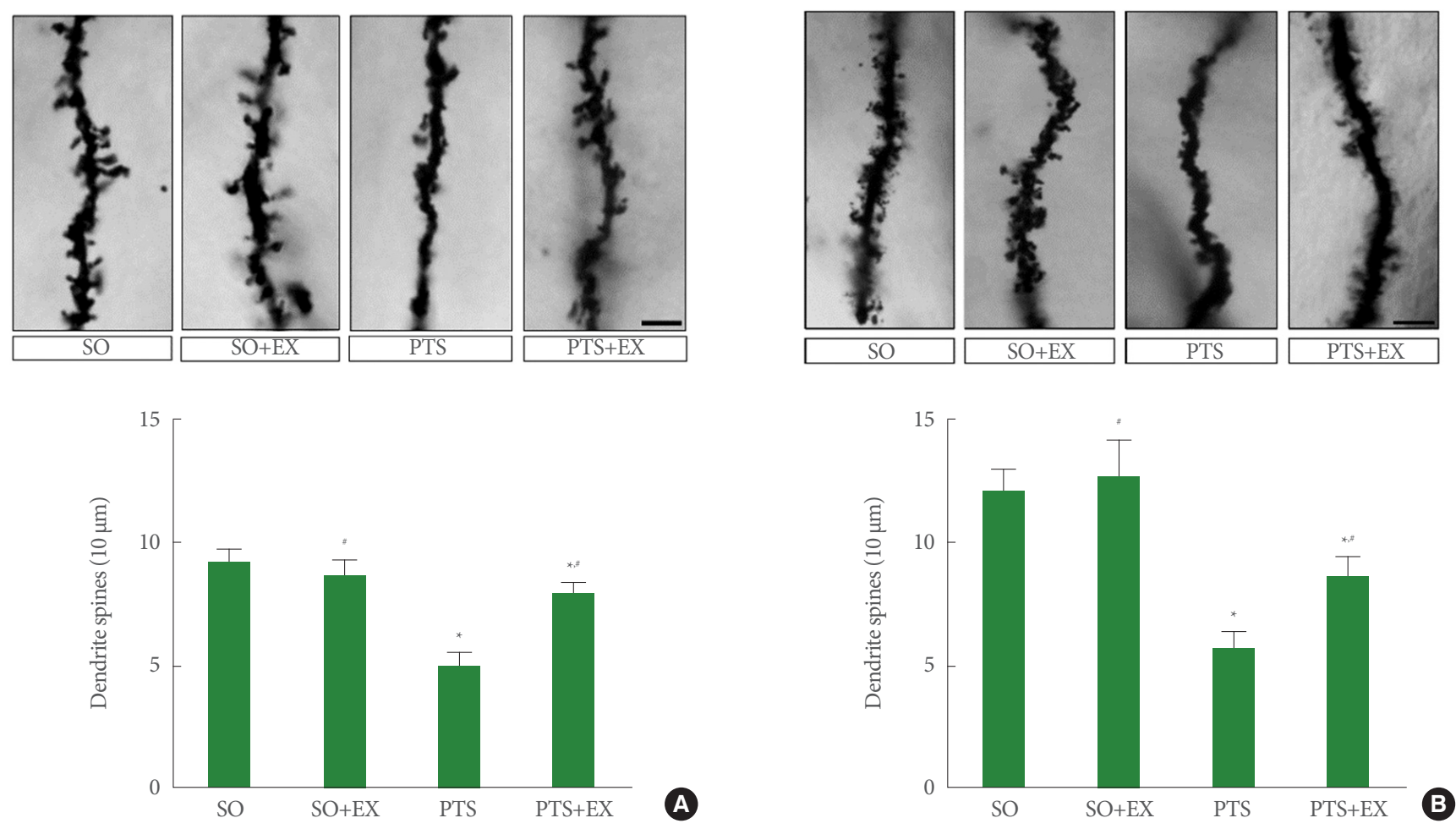

Fig. 4. Dendritic spines in the peri-infarct cortex (A) and the hippocampus (B). SO, sham-operation group; SO+EX, sham-operation and exercise group; PTS, photothrombotic stroke-induction group; PTS+EX, photothrombotic stroke-induction and exercise group. Data show mean \pm standard error of the mean. ${ }^{*} \mathrm{P}<0.05$ compared with the sham-operation group. ${ }^{*} \mathrm{P}<0.05$ compared with the photothrombotic stroke-induction group. 

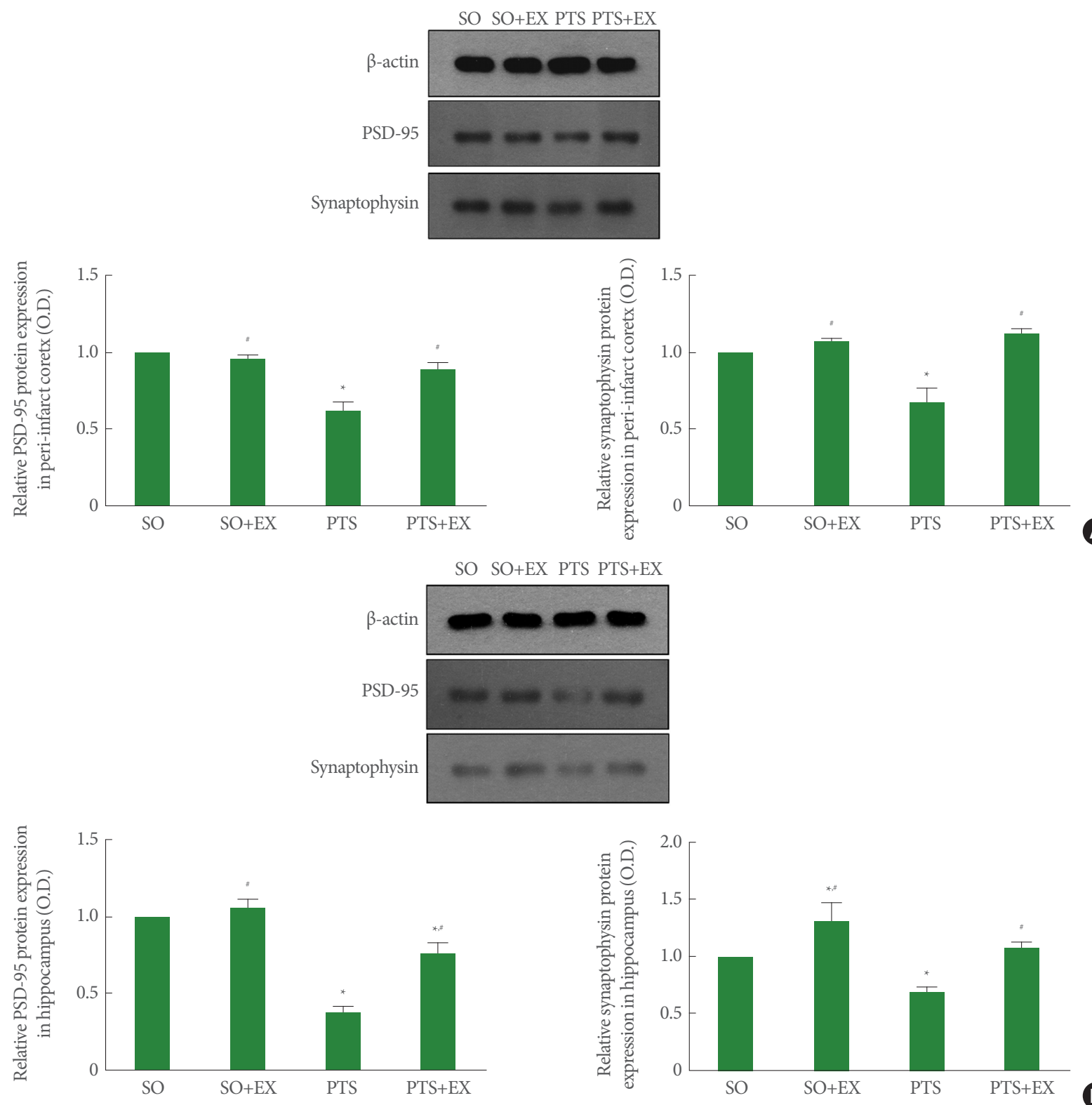

B

Fig. 5. Expression of postsynaptic density protein 95 (PSD-95) and synaptophysin in the peri-infarct cortex (A) and hippocampus (B). SO, sham-operation group; SO+EX, sham-operation and exercise group; PTS, photothrombotic stroke-induction group; PTS+EX, photothrombotic stroke-induction and exercise group. Data show mean \pm standard error of the mean. ${ }^{\star} \mathrm{P}<0.05$ compared with the sham-operation group. ${ }^{*} \mathrm{P}<0.05$ compared with the photothrombotic stroke-induction group.

compared with the photothrombotic stroke-induction group $(\mathrm{P}<0.05)$.

\section{Neurogenesis}

Fig. 6 shows the number of DCX-positive cells (panel A) and BrdU-positive cells (panel B) in the hippocampal dentate gyrus. The photothrombotic stroke reduced the number of DCX-positive cells in the hippocampal dentate gyrus compared with the shamoperation group $(\mathrm{P}<0.05)$, while treadmill exercise enhanced the number of DCX-positive cells in the hippocampus compared with the photothrombotic stroke-induction group $(\mathrm{P}<0.05)$. The photothrombotic stroke reduced the number of BrdU-positive cells in the hippocampal dentate gyrus compared with the shamoperation group $(\mathrm{P}<0.05)$, while treadmill exercise enhanced the 

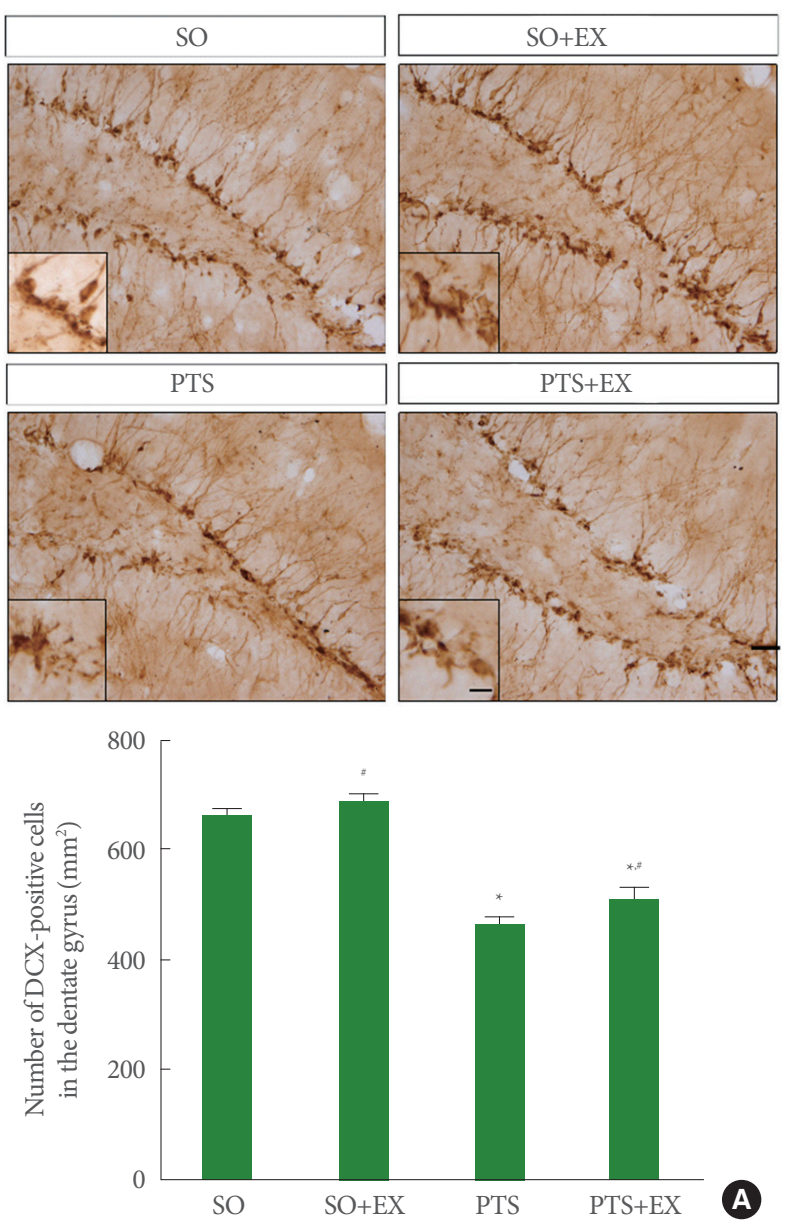
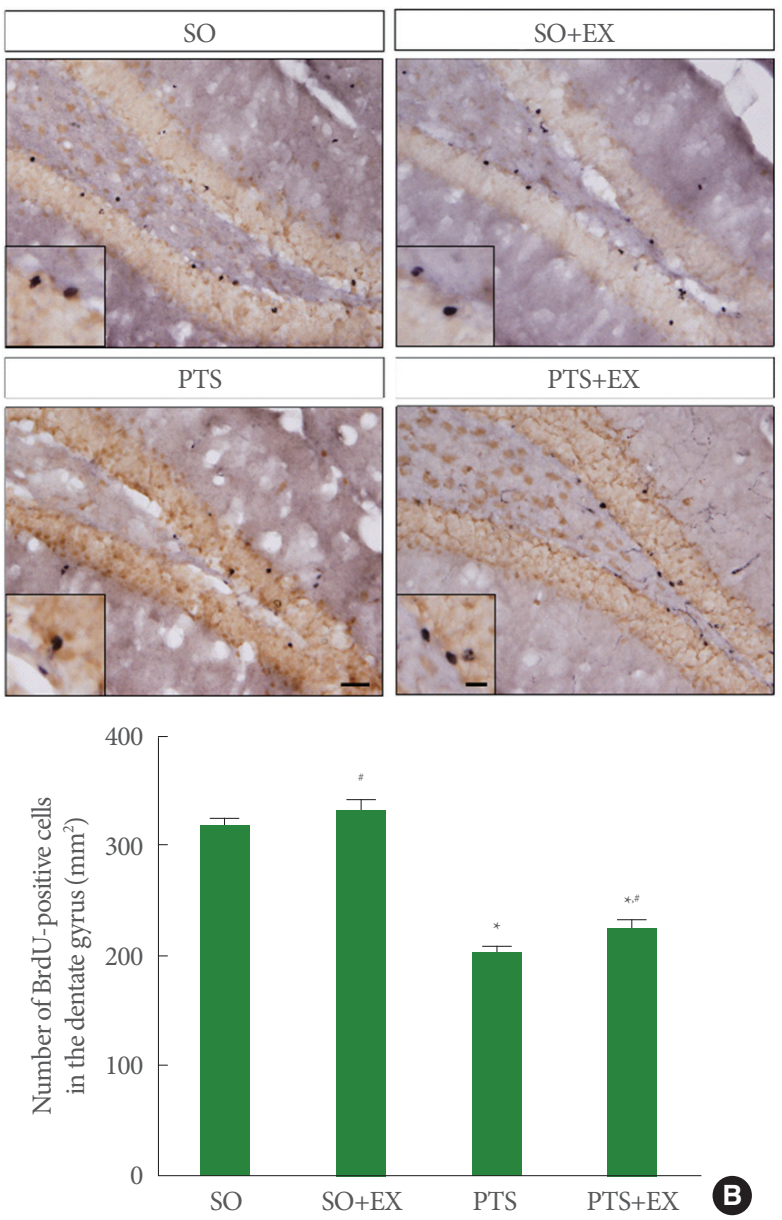

Fig. 6. Effect of treadmill exercise on the number of doublecortin (DCX)-positive cells (A) and 5-bromo-2'-deoxyuridine (BrdU)positive cells (B) in the hippocampal dentate gyrus. SO, sham-operation group; SO+EX, sham-operation and exercise group; PTS, photothrombotic stroke-induction group; PTS+EX, photothrombotic stroke-induction and exercise group. Insets are at higher magnification $(10 \mu \mathrm{m})$. Data show mean \pm standard error of the mean. ${ }^{*} \mathrm{P}<0.05$ compared with the sham-operation group. ${ }^{*} \mathrm{P}<0.05$ compared with the photothrombotic stroke-induction group.

number of BrdU-positive cells in the hippocampus compared with the photothrombotic stroke-induction group $(\mathrm{P}<0.05)$.

\section{BDNF and TrkB Expression}

Fig. 7 shows the relative expression of BDNF and TrkB in the hippocampus. Photothrombotic stroke reduced the relative expression of BDNF and TrkB in the hippocampus compared with the sham-operation group $(\mathrm{P}<0.05)$, while treadmill exercise enhanced the relative expression of BDNF and TrkB compared with the photothrombotic stroke-induction group $(\mathrm{P}<0.05)$.

\section{DISCUSSION}

Thrombotic stroke eventually leads to neurological deficits and cognitive impairments [22]. Stroke is a common cause of movement-related disorders, such as defects of balance and coordination [23]. Photothrombotic stroke is produced by occlusion of small brain vessels, and this model is widely used to evaluate the underlying mechanism of cerebral ischemic stroke [4]. Rota-rod test and foot fault test are mainly used to determine motor function such as motor adjustment and balance [24]. In the results of this study, induction of photothrombotic stroke shortened the latency to fall of the rota-rod test and increased the foot fault score of the foot fault test. On the contrary, treadmill exercise increased the latency to fall and decreased the foot fault score in photothrombotic stroke mice. Exercise improved neurological deficits in the stroke animal model $[25,26]$. 

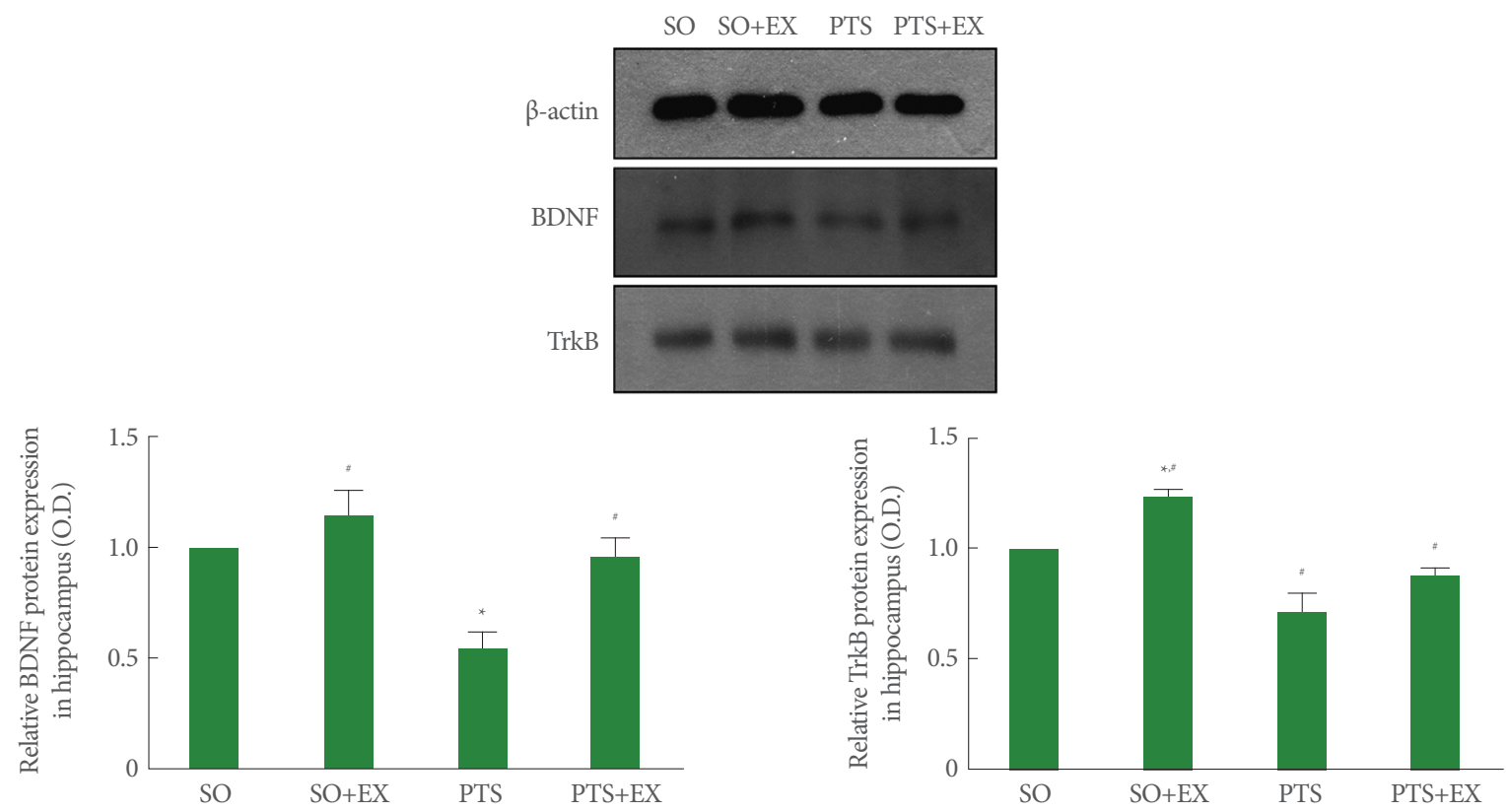

Fig. 7. Effect of treadmill exercise on the brain-derived neurotrophic factor (BDNF) and tyrosine kinase B receptor (TrkB) in the hippocampus. SO, sham-operation group; SO+EX, sham-operation and exercise group; PTS, photothrombotic stroke-induction group; $\mathrm{PTS}+\mathrm{EX}$, photothrombotic stroke-induction and exercise group. Data show mean \pm standard error of the mean. ${ }^{\star} \mathrm{P}<0.05$ compared with the sham-operation group. ${ }^{*} \mathrm{P}<0.05$ compared with the photothrombotic stroke-induction group.

Ischemic stroke causes deficits in learning ability and memory [22]. In the results of this study, the latency in the stepthrough avoidance task was shorted by induction of photothrombotic stroke. On the contrary, treadmill exercise improved the photothrombotic stroke-induced short-term memory impairments. Exercise is known to improve short-term memory and cognitive function [15,27].

Photothrombotic ischemia is known to cause severe massive tissue infarction and damage [17]. The amount of cortical infarction lesions is closely related to lack of motor function or cognitive impairments [20]. In the present study, infarct size was increased by induction of photothrombotic stroke, on the contrary, treadmill exercise suppressed infarct size in photothrombotic stroke mice.

Function recovery after stroke can be triggered by synaptic remodeling and neuroplasticity [28]. Loss of dendritic spines after ischemic insult was reported [29]. Dendritic spines play an important role in cognitive and motor function as well as memory formation. Memory formation increases dendritic spine density, which reflects improved learning and memory ability [30]. In the results of this study, the number of dendritic spines in the peri-infarct cortex and the hippocampal dentate gyrus was reduced by induction of photothrombotic stroke. On the contrary, treadmill exercise increased the number of dendritic spines in the peri-infarct cortex and the hippocampal dentate gyrus.

Synaptogenesis acts a major role in learning and memory process [31]. Synaptophysin and PSD-95 are important markers that play an important role in the synaptic formation and reconstruction [7]. The expression level of PSD-95 and synaptophysin was decreased in the hippocampus by cerebral ischemic stroke [32,33]. In the results of this study, expression of PSD-95 and synaptophysin was reduced in the peri-infarct cortex and hippocampus after induction of photothrombotic stroke. On the contrary, treadmill exercise increased expression of PSD-95 and synaptophysin in the peri-infarct cortex and hippocampus after photothrombotic stroke.

Reduced neurogenesis of the hippocampal dentate gyrus was closely related to loss of learning and memory function [10,18,21]. In the results of this study, the number of BrdU-positive and DCX-positive cells was reduced after induction of photothrombotic stroke. On the contrary, treadmill exercise enhanced the number of BrdU-positive and DCX-positive cells after photothrombotic stroke. Exercise can promote neurogenesis in the hippocampal dentate gyrus [21], and this neuronal production is associated with the relief of short-term memory impairments 
$[15,16]$.

Hippocampal BDNF is implicated in neuronal survival, alteration of synaptic strength, and memory capacity $[18,34]$. BDNF-TrkB signaling mediated hippocampal neurogenesis, and enhanced BDNF and TrkB signaling was closely related to the improvement of hippocampal-dependent memory by exercise $[13,21]$. In the results of this study, the expression of hippocampal BDNF and TrkB was decreased after induction of photothrombotic stroke. On the contrary, treadmill exercise enhanced hippocampal BDNF and TrkB expression after photothrombotic stroke. High BDNF and TrkB expression increases neurogenesis caused by treadmill exercise.

In this study, treadmill exercise improved motor function and short-term memory through increasing synaptic proteins and neurogenesis in photothrombotic stroke mice. The conclusion of this experiment suggests that exercise also has a positive effect on genitourinary diseases. Based on the present results, treadmill exercise can be used as an effective treatment modality to improve brain function associated with stroke.

\section{AUTHOR CONTRIBUTION STATEMENT}

- Conceptualization: $M H, M K$
- Formal Analysis: $M H, M K$
- Investigation: TWK, SSP
- Methodology: $M K K, Y H P, Y H S$
- Project Administration: MSS
- Writing - Original Draft: MSS
- Writing - Review \& Editing: MSS

\section{REFERENCES}

1. Shah FA, Li T, Kury LTA, Zeb A, Khatoon S, Liu G, et al. Pathological comparisons of the hippocampal changes in the transient and permanent middle cerebral artery occlusion rat Models. Front Neurol 2019;10:1178.

2. Kunz A, Iadecola C. Cerebral vascular dysregulation in the ischemic brain. Handb Clin Neurol 2009;92:283-305.

3. Donnini S, Solito R, Monti M, Balduini W, Carloni S, Cimino M, et al. Prevention of ischemic brain injury by treatment with the membrane penetrating apoptosis inhibitor, TAT-BH. Cell Cycle 2009; 8:1271-8.

4. Uzdensky AB. Photothrombotic stroke as a model of ischemic stroke. Transl Stroke Res 2018;9:437-51.

5. Hermann DM and Chopp M. Promoting brain remodelling and plasticity for stroke recovery: Therapeutic promise and potential pitfalls of clinical translation. Lancet Neurol 2012;11:369-80.

6. Mayford M, Siegelbaum SA, Kandel ER. Synapses and memory storage. Cold Spring Harb Perspect Biol 2012;4: a005751.

7. Shilpa BM, Bhagya V, Harish G, Srinivas Bharath MM, Shankaranarayana Rao BS. Environmental enrichment ameliorates chronic immobilisation stress-induced spatial learning deficits and restores the expression of BDNF, VEGF, GFAP and glucocorticoid receptors. Prog Neuropsychopharmacol Biol Psychiatry 2017;76: 88-100.

8. De Camilli P, Vitadello M, Canevini MP, Zanoni R, Jahn R, Gorio A. The synaptic vesicle proteins synapsin I and synaptophysin (protein P38) are concentrated both in efferent and afferent nerve endings of the skeletal muscle. J Neurosci 1988;8:1625-31.

9. Deng W, Aimone JB, Gage FH. New neurons and new memories: how does adult hippocampal neurogenesis affect learning and memory? Nat Rev Neurosci 2010;11:339-50.

10. Rola R, Raber J, Rizk A, Otsuka S, VandenBerg SR, Moreherdt DR, et al. Radiation-induced impairment of hippocampal neurogenesis is associated with cognitive deficits in young mice. Exp Neurol 2004;188:316-30.

11. Bothwell M. NGF, BDNF, NT3, and NT4. Handb Exp Pharmacol 2014;220:3-15.

12. Liu PZ, Nusslock R. Exercise-mediated neurogenesis in the hippocampus via BDNF. Front Neurosci 2018;12:52.

13. Park SS, Shin MS, Park HS, Kim TW, Kim CJ, Lim BV. Treadmill exercise ameliorates nicotine withdrawal-induced symptoms. J Exerc Rehabil 2019;15:383-91.

14. Lee SS, Kim CJ, Shin MS, Lim BV. Treadmill exercise ameliorates memory impairment through ERK-Akt-CREB-BDNF signaling pathway in cerebral ischemia gerbils. J Exerc Rehabil 2020;16:4957.

15. Lee JM, Baek SS, Kim TW, Park HS, Park SS, Park JM, et al. Preischemic treadmill exercise improves short-term memory by inhibiting hypoperfusion-induced disruption of blood-brain barrier after bilateral common carotid arteries occlusion. J Exerc Rehabil 2019;15:370-6.

16. Ding Y, Li J, Luan X, Ding YH, Lai Q, Rafols JA, et al. Exercise preconditioning reduces brain damage in ischemic rats that may be associated with regional angiogenesis and cellular overexpression of neurotrophin. Neuroscience 2004;124:583-91.

17. Shin TK, Kang MS, Lee HY, Seo MS, Kim SG, Kim CD, et al. Fluoxetine and sertraline attenuate postischemic brain injury in mice. Korean J Physiol Pharmacol 2009;13:257-63.

18. Kim SE, Ko IG, Ji ES, Jin JJ, Hwang L, Kim SH, et al. Treadmill ex- 
ercise alleviates circadian rhythm disruption-induced memory deficits by activation of glucocorticoid receptor and brain-derived neurotrophic factor-dependent pathway. Int Neurourol J 2019; 23:S40-9.

19. Lee JM, Kim TW, Park SS, Han JH, Shin MS, Lim BV, et al. Treadmill exercise improves motor function by suppressing Purkinje cell loss in Parkinson disease rats. Int Neurourol J 2018;22:S147-55.

20. Shelton SB, Pettigrew DB, Hermann AD, Zhou W, Sullivan PM, Crutcher KA, et al. A simple, efficient tool for assessment of mice after unilateral cortex injury. J Neurosci Methods 2008;168:431-42.

21. Cho JW, Jung SY, Lee SW, Lee SJ, Seo TB, Kim YP, et al. Treadmill exercise ameliorates social isolation-induced depression through neuronal generation in rat pups. J Exerc Rehabil 2017;13:627-33.

22. Zheng G, Zheng Y, Xiong Z, Ye B, Tao J, Chen L. Effect of Baduanjin exercise on cognitive function in patients with post-stroke cognitive impairment: study protocol for a randomised controlled trial. BMJ Open 2018;8:e020954.

23. Langhorne P, Coupar F, Pollock A. Motor recovery after stroke: a systematic review. Lancet Neurol 2009;8:741-54.

24. Schaar KL, Brenneman MM, Savitz SI. Functional assessments in the rodent stroke model. Exp Transl Stroke Med 2010;2:13.

25. Naderi S, Alimohammadi R, Hakimizadeh E, Roohbakhsh A, Shamsizadeh A, Allahtavakoli M. The effect of exercise preconditioning on stroke outcome in ovariectomized mice with permanent middle cerebral artery occlusion. Can J Physiol Pharmacol 2018; 96:287-94.
26. Liu N, Huang H, Lin F, Chen A, Zhang Y, Chen R, et al. Effects of treadmill exercise on the expression of netrin- 1 and its receptors in rat brain after cerebral ischemia. Neuroscience 2011;194:349-58.

27. Okonkwo O, van Praag H. Exercise effects on cognitive function in humans. Brain Plast 2019;5:1-2.

28. Murphy TH, Corbett D. Plasticity during stroke recovery: from synapse to behaviour. Nat Rev Neurosci 2009;10:861-72.

29. Lin R, Wu Y, Tao J, Chen B, Chen J, Zhao C, et al. Electroacupuncture improves cognitive function through Rho GTPases and enhances dendritic spine plasticity in rats with cerebral ischemia-reperfusion. Mol Med Rep 2016;13:2655-60.

30. Halpain S, Spencer K, Graber S. Dynamics and pathology of dendritic spines. Prog Brain Res 2005;147:29-37.

31. Nelson TJ, Alkon DL. Molecular regulation of synaptogenesis during associative learning and memory. Brain Res 2015;1621:239-51.

32. Wang S, Yuan Y, Xia W, Li F, Huang Y, Zhou Y, et al. Neuronal apoptosis and synaptic density in the dentate gyrus of ischemic rats response to chronic mild stress and the effects of Notch signaling. PLoS One 2012;7:e42828.

33. Yan BC, Park JH, Ahn JH, Lee JC, Won MH, Kang IJ. Postsynaptic density protein (PSD)-95 expression is markedly decreased in the hippocampal CA1 region after experimental ischemia-reperfusion injury. J Neurol Sci 2013;330:111-6.

34. Alonso M, Medina JH, Pozzo-Miller L. ERK1/2 activation is necessary for BDNF to increase dendritic spine density in hippocampal CA1 pyramidal neurons. Learn Mem 2004;11:172-8. 